\title{
AXIOMATIC DIFFERENTIAL GEOMETRY II-1 - VECTOR FIELDS
}

\author{
HIROKAZU NISHIMURA
}

\begin{abstract}
In our previous paper entitled "Axiomatic differential geometry I - towards model categories of differential geometry", we have given a category-theoretic framework of differential geometry. As the first part of our series of papers concerned with differential-geometric developments within the above axiomatic scheme, this paper is devoted to vector fields. The principal result is that the totality of vector fields on a microlinear and Weil exponential object forms a Lie algebra.
\end{abstract}

\section{INTRODUCTION}

In [4] we have given a skeleton of our axiomatic approach to differential geometry. This paper, concerned with vector fields, is the first part of our theoretical developments within the axiomatic framework. Subsequent papers deal with differential forms, the Frölicher-Nijenhuis calculus, jet bundles, connections and so on.

In Section 2, we develop a convenient system of locutions in speaking of Weil algebras. Since we are no longer allowed to speak elementwise in our general context, we have to learn how to express "the tangent space is a linear space", to say nothing of how to prove it, which will be done in Section 3. The principal result of Section 4 is that the totality of vector fields forms a Lie algebra.

\section{Preliminaries}

\subsection{Weil Algebras and Infinitesimal Objects}

Let $k$ be a commutative ring. As in our previous paper, we denote by $\mathbf{W e i l}_{k}$ the category of Weil $k$-algebras. Roughly speaking, each Weil $k$-algebra corresponds to an infinitesimal object in the shade. By way of example, the Weil algebra $k[X] /\left(X^{2}\right)$ (=the quotient ring of the polynomial ring $k[X]$ of an indeterminate $X$ over $k$ modulo the ideal $\left(X^{2}\right)$ generated by $\left.X^{2}\right)$ corresponds to the infinitesimal object of first-order nilpotent infinitesimals, while the Weil algebra $k[X] /\left(X^{3}\right)$ corresponds to the infinitesimal object of second-order nilpotent infinitesimals. Although an infinitesimal object is undoubtedly imaginary in our real world, as has harassed both mathematicians and philosophers of the 17th and the 18th centuries such as extravagantly skeptical philosopher Berkley (because mathematicians at that time preferred to talk infinitesimal objects as if they were real entities), each Weil algebra yields its corresponding Weil functor or Weil prolongation in our real

$M S C$ (2010): primary 53Z99,18F99.

Keywords: axiomatic differential geometry, Weil functor, vector field, convenient category. 
world. By way of example, the Weil algebra $k[X] /\left(X^{2}\right)$ yields the tangent bundle functor as its corresponding Weil functor. Weil functors are major players in our axiomatic approach.

Synthetic differential geometry (usually abbreviated to SDG), which is a kind of differential geometry with a cornucopia of nilpotent infinitesimals, was forced to invent its models, in which nilpotent infinitesimals were transparently visible. For a standard textbook on SDG, the reader is referred to [2], while he or she is referred to [1] for the model theory of SDG. Although we do not get involved in SDG herein, we will exploit locutions in terms of infinitesimal objects so as to make the paper highly readable. Thus we prefer to write $\mathcal{W}_{D}$ and $\mathcal{W}_{D_{2}}$ in place of $k[X] /\left(X^{2}\right)$ and $k[X] /\left(X^{3}\right)$ respectively, where $D$ stands for the infinitesimal object of first-order nilpotent infinitesimals, and $D_{2}$ stands for the infinitesimal object of second-order nilpotent infinitesimals. More generally, given a natural number $n$, we denote by $D_{n}$ the infinitesimal object corresponding to the Weil $k$-algebra $k[X] /\left(X^{n+1}\right)$. Obviously we have $D_{1}=D$. Even more generally, given natural numbers $m, n$, we denote by $D(m)_{n}$ the infinitesimal object corresponding to the Weil algebra $k\left[X_{1}, \ldots, X_{m}\right] / I$, where $I$ is the ideal generated by $X_{i_{1}} \ldots X_{i_{n+1}}$ 's with $i_{1}, \ldots, i_{n+1}$ being integers such that $1 \leq i_{1}, \ldots, i_{n+1} \leq m$. Therefore we have $D(1)_{n}=D_{n}$, while we write $D(m)$ for $D(m)_{1}$. We will write $\mathcal{W}_{d \in D_{2} \mapsto d^{2} \in D}$, by way of example, for the homomorphim of Weil algebras $k[X] /\left(X^{2}\right) \rightarrow k[X] /\left(X^{3}\right)$ induced by the homomorphism $X \rightarrow X^{2}$ of the polynomial ring $k[X]$ to itself. Such locutions are justifiable, because the category Weil W $_{k}$ of Weil $k$-algebras in the real world and the category of infinitesimal objects in the shade are dual to each other in a sense. Thus we have a contravariant functor $\mathcal{W}$ from the category of infinitesimal objects in the shade to the category of Weil algebras in the real world, yielding a contravariant equivalence between the two categories.

\subsection{Assumptions}

We fix a DG-category $(\mathcal{K}, \mathbb{R}, \mathbf{T}, \alpha)$ in the sense of [4], while $M$ is a microlinear and Weil exponentiable object in $\mathcal{K}$.

\section{TANGEnt BundLES}

Proposition 3.1. If $M$ is a microlinear object in $\mathcal{K}$, then we have

$$
M \otimes \mathcal{W}_{D(2)}=\left(M \otimes \mathcal{W}_{D}\right) \times_{M}\left(M \otimes \mathcal{W}_{D}\right)
$$

Proof. We have the following pullback diagram of Weil $k$-algebras:

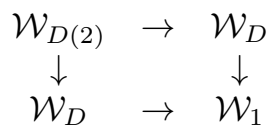

where the left vertical arrow is

$$
\mathcal{W}_{d \in D \mapsto(d, 0) \in D(2)}
$$

while the upper horizontal arrow is

$$
\mathcal{W}_{d \in D \mapsto(0, d) \in D(2)} .
$$


The above pullback diagram naturally gives rise to the following pullback diagram because of the microlinearity of $M$ :

$$
\begin{array}{ccc}
M \otimes \mathcal{W}_{D(2)} & \rightarrow & M \otimes \mathcal{W}_{D} \\
\downarrow & & \downarrow \\
M \otimes \mathcal{W}_{D} & \rightarrow & M \otimes \mathcal{W}_{1}=M .
\end{array}
$$

This completes the proof.

Corollary 3.2. The canonical projection $\tau_{\mathcal{W}_{D(2)}}(M): M \otimes \mathcal{W}_{D(2)} \rightarrow M$ is a product of two copies of $\tau_{\mathcal{W}_{D}}(M): M \otimes \mathcal{W}_{D} \rightarrow M$ in the slice category $\mathcal{K} / M$.

Now we are in a position to define basic operations on $\tau_{\mathcal{W}_{D}}(M): M \otimes \mathcal{W}_{D} \rightarrow M$ in the slice category $\mathcal{K} / M$ so as to make it a $k$-module.

(1) The addition is defined by

$$
\operatorname{id}_{M} \otimes \mathcal{W}_{+_{D}}: M \otimes \mathcal{W}_{D(2)} \rightarrow M \otimes \mathcal{W}_{D}
$$

where the fabulous mapping $+_{D}: D \rightarrow D(2)$ is

$$
+_{D}: d \in D \mapsto(d, d) \in D(2) .
$$

(2) The identity with respect to the above addition is defined by

$$
\operatorname{id}_{M} \otimes \mathcal{W}_{0_{D}}: M=M \otimes \mathcal{W}_{1} \rightarrow M \otimes \mathcal{W}_{D}
$$

where the fabulous mapping $0_{D}: D \rightarrow 1$ is the unique mapping.

(3) The inverse with respect to the above addition is defined by

$$
\operatorname{id}_{M} \otimes \mathcal{W}_{-D}: M \otimes \mathcal{W}_{D} \rightarrow M \otimes \mathcal{W}_{D}
$$

where the fabulous mapping $-{ }_{D}: D \rightarrow D$ is

$$
-{ }_{D}: d \in D \mapsto-d \in D .
$$

(4) The scalar multiplication by a scalar $\xi \in k$ is defined by

$$
\operatorname{id}_{M} \otimes \mathcal{W}_{\xi_{D}}: M \otimes \mathcal{W}_{D} \rightarrow M \otimes \mathcal{W}_{D}
$$

where the fabulous mapping $\xi_{D}: D \rightarrow D$ is

$$
\xi_{D}: d \in D \mapsto \xi d \in D .
$$

Now we have

Theorem 3.3. The canonical projection $\tau_{\mathcal{W}_{D}}(M): M \otimes \mathcal{W}_{D} \rightarrow M$ is a $k$ module in the slice category $\mathcal{K} / M$.

Proof.

(1) The associativity of the addition follows from the following commutative diagram:

$$
\begin{array}{ccccc} 
& \multicolumn{4}{c}{\operatorname{id}_{M} \otimes \mathcal{W}_{\epsilon_{23}}} \\
\operatorname{id}_{M} \otimes \mathcal{W}_{\epsilon_{12}} & \downarrow & \rightarrow & M \otimes \mathcal{W}_{D(2)} & \\
& M \otimes \mathcal{W}_{D(2)} & & \downarrow & \operatorname{id}_{M} \otimes \mathcal{W}_{+_{D}} \\
& & \rightarrow & M \otimes \mathcal{W}_{D} & \\
\operatorname{id}_{M} \otimes \mathcal{W}_{+_{D}} & &
\end{array}
$$

where the fabulous mapping $\epsilon_{23}: D(2) \rightarrow D(3)$ is

$$
\left(d_{1}, d_{2}\right) \in D(2) \mapsto\left(d_{1}, d_{1}, d_{2}\right) \in D(3)
$$


while the fabulous mapping $\epsilon_{12}: D(2) \rightarrow D(3)$ is

$$
\left(d_{1}, d_{2}\right) \in D(2) \mapsto\left(d_{1}, d_{2}, d_{2}\right) \in D(3) .
$$

(2) The commutativity of the addition follows readily from the commutative diagram

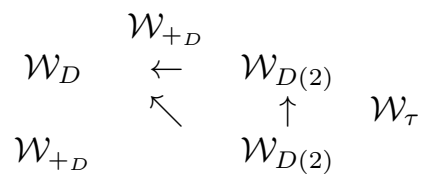

where the fabulous mapping $\tau: D(2) \rightarrow D(2)$ is

$$
\left(d_{1}, d_{2}\right) \in D(2) \mapsto\left(d_{2}, d_{1}\right) \in D(2) .
$$

(3) To see that the identity defined above really plays the identity with respect to the above addition, it suffices to note that the composition of the following two fabulous mappings

$$
\begin{aligned}
& d \in D \mapsto(d, 0) \in D(2) \\
& \left(d_{1}, d_{2}\right) \in D(2) \mapsto d_{1} \in D
\end{aligned}
$$

in order is the identity mapping of $D$, while the composition of the following two fabulous mappings

$$
\begin{aligned}
& d \in D \mapsto(0, d) \in D(2) \\
& \left(d_{1}, d_{2}\right) \in D(2) \mapsto d_{1} \in D
\end{aligned}
$$

in order is the constant mapping

$$
d \in D \mapsto 0 \in D .
$$

(4) To see that the addition of scalars distributes with respect to the scalar multiplication, it suffices to note that, for any $\xi_{1}, \xi_{2} \in k$, the composition of the following two fabulous mappings

$$
\begin{aligned}
& d \in D \mapsto(d, 0) \in D(2) \\
& \left(d_{1}, d_{2}\right) \in D(2) \mapsto \xi_{1} d_{1}+\xi_{2} d_{2} \in D
\end{aligned}
$$

in order is the mapping

$$
d \in D \mapsto \xi_{1} d_{1} \in D
$$

and the composition of the two fabulous mappings

$$
\begin{aligned}
& d \in D \mapsto(0, d) \in D(2) \\
& \left(d_{1}, d_{2}\right) \in D(2) \mapsto \xi_{1} d_{1}+\xi_{2} d_{2} \in D
\end{aligned}
$$

in order is the mapping

$$
d \in D \mapsto \xi_{2} d_{2} \in D
$$

while the composition of the two fabulous mappings

$$
\begin{aligned}
& d \in D \mapsto(d, d) \in D(2) \\
& \left(d_{1}, d_{2}\right) \in D(2) \mapsto \xi_{1} d_{1}+\xi_{2} d_{2} \in D
\end{aligned}
$$


in order is no other than the mapping

$$
d \in D \mapsto\left(\xi_{1}+\xi_{2}\right) d \in D .
$$

(5) To see that the addition of vectors distributes with respect to the scalar multiplication, it suffices to note that, for any $\xi \in k$, the composition of the two fabulous mappings

$$
\begin{aligned}
& d \in D \mapsto(d, 0) \in D(2) \\
& \left(d_{1}, d_{2}\right) \in D(2) \mapsto\left(\xi d_{1}, \xi d_{2}\right) \in D(2)
\end{aligned}
$$

in order is the composition of the two fabulous mappings

$$
\begin{aligned}
& d \in D \mapsto \xi d \in D \\
& d \in D \mapsto(d, 0) \in D(2)
\end{aligned}
$$

in order, and the composition of the two fabulous mappings

$$
\begin{aligned}
& d \in D \mapsto(0, d) \in D(2) \\
& \left(d_{1}, d_{2}\right) \in D(2) \mapsto\left(\xi d_{1}, \xi d_{2}\right) \in D(2)
\end{aligned}
$$

in order is the composition of the two fabulous mappings

$$
\begin{aligned}
& d \in D \mapsto \xi d \in D \\
& d \in D \mapsto(0, d) \in D(2)
\end{aligned}
$$

in order, while the composition of the two fabulous mappings

$$
\begin{aligned}
& d \in D \mapsto(d, d) \in D(2) \\
& \left(d_{1}, d_{2}\right) \in D(2) \mapsto\left(\xi d_{1}, \xi d_{2}\right) \in D(2)
\end{aligned}
$$

in order is no other than the composition of the two fabulous mappings

$$
\begin{aligned}
& d \in D \mapsto \xi d \in D \\
& d \in D \mapsto(d, d) \in D(2)
\end{aligned}
$$

in order.

(6) The verification of the other axioms for being a $k$-module can safely be left to the reader.

Remark 3.4. Given a morphims $f: N \rightarrow M$ in $\mathcal{K}$, the pullback functor $f^{*}: \mathcal{K} / M \rightarrow \mathcal{K} / N$ is left exact ( preserving finite products, in particular), so that

$$
f^{*}\left(\begin{array}{c}
M \otimes \mathcal{W}_{D} \\
\tau_{\mathcal{W}_{D}}(M) \\
\downarrow \\
M
\end{array}\right)
$$

is a $k$-module in $\mathcal{K} / N$. In case that $N=1$, we have $\mathcal{K} / 1=\mathcal{K}$ yielding the notion of the tangent space $\left(M \otimes \mathcal{W}_{D}\right)_{x}$ of $M$ at a point $x: 1 \rightarrow M$. 


\section{The Lie Algebra of Vector Fields}

The totality of vector fields on $M$ can be delineated in two distinct ways. First we must fix our notation.

Notation 4.1. We write $\left(\left(M \otimes \mathcal{W}_{D}\right)^{M}\right)_{\operatorname{id}_{M}}$ for the pullback of

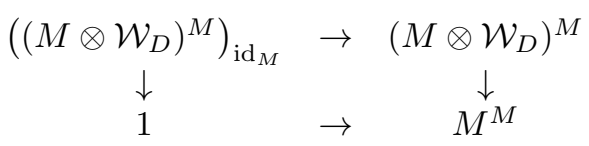

where the right vertical arrow is

$$
\left(\tau_{\mathcal{W}_{D}}(M)\right)^{M}:\left(M \otimes \mathcal{W}_{D}\right)^{M} \rightarrow M^{M},
$$

while the bottom horizontal arrow is the exponential transpose of

$$
\operatorname{id}_{M}: M=1 \times M \rightarrow M \text {. }
$$

Notation 4.2. We write $\left(M^{M} \otimes \mathcal{W}_{D}\right)_{\mathrm{id}_{M}}$ for the pullback of

$$
\begin{array}{ccc}
\left(M^{M} \otimes \mathcal{W}_{D}\right)_{\operatorname{id}_{M}} & \rightarrow & M^{M} \otimes \mathcal{W}_{D} \\
\downarrow & & \downarrow \\
1 & \rightarrow & M^{M}
\end{array}
$$

where the right vertical arrow is

$$
\tau_{M^{M}}: M^{M} \otimes \mathcal{W}_{D} \rightarrow M^{M}
$$

while the bottom horizontal arrow is the exponential transpose of

$$
\operatorname{id}_{M}: M=1 \times M \rightarrow M \text {. }
$$

Theorem 4.3. The object $\left(\left(M \otimes \mathcal{W}_{D}\right)^{M}\right)_{\mathrm{id}_{M}}$ can naturally be identified with the object $\left(M^{M} \otimes \mathcal{W}_{D}\right)_{\mathrm{id}_{M}}$.

Proof. It suffices to note that the diagram

$$
\begin{array}{ccc}
\left(M \otimes \mathcal{W}_{D}\right)^{M} & = & M^{M} \otimes \mathcal{W}_{D} \\
\searrow & M^{M} & \swarrow
\end{array}
$$

is commutaive, where the right slant arrow is $\tau_{\mathcal{W}_{D}}\left(M^{M}\right): M^{M} \otimes \mathcal{W}_{D} \rightarrow M^{M}$, while the left slant arrow is $\left(\tau_{\mathcal{W}_{D}}(M)\right)^{M}:\left(M \otimes \mathcal{W}_{D}\right)^{M} \rightarrow M^{M}$.

Remark 4.4. Thus the totality of vector fields on $M$ is represented by $\left(M^{M} \otimes\right.$ $\left.\mathcal{W}_{D}\right)_{\operatorname{id}_{M}}$ as well as by $\left(\left(M \otimes \mathcal{W}_{D}\right)^{M}\right)_{\operatorname{id}_{M}}$. The first viewpoint, which reckons vector fields as the tangent space to $M$ at the identitity transformation, is preferred in this paper, while the second viewpoint, which regards vector fields on $M$ as sections of the tangent bundle $\tau_{\mathcal{W}_{D}}(M): M \otimes \mathcal{W}_{D} \rightarrow M$, has been orthodox in conventional differential geometry.

Notation 4.5. We write $\operatorname{ass}_{M}: M^{M} \times M^{M} \rightarrow M^{M}$ for the morphism obtained as the exponential transpose of the composition of

$$
\mathrm{ev}_{M} \times \operatorname{id}_{M^{M}}: M \times M^{M} \times M^{M}=\left(M \times M^{M}\right) \times M^{M} \rightarrow M \times M^{M}
$$


and

$$
\mathrm{ev}_{M}: M \times M^{M} \rightarrow M
$$

where $\mathrm{ev}_{M}: M \times M^{M} \rightarrow M$ stands for the evaluation morphism. Similarly, we write $\overline{\operatorname{ass}}_{M}: M^{M} \times M^{M} \rightarrow M^{M}$ for the morphism obtained as the exponential transpose of the composition of

$$
\begin{aligned}
& \operatorname{id}_{M^{M}} \times \operatorname{ev}_{M}: M \times M^{M} \times M^{M}=M^{M} \times M \times M^{M}=M^{M} \times\left(M \times M^{M}\right) \\
\rightarrow & M^{M} \times M=M \times M^{M}
\end{aligned}
$$

and

$$
\operatorname{ev}_{M}: M \times M^{M} \rightarrow M .
$$

Notation 4.6. We write $\operatorname{Ass}_{M}^{m, n}:\left(M^{M} \otimes \mathcal{W}_{D^{m}}\right) \times\left(M^{M} \otimes \mathcal{W}_{D^{n}}\right) \rightarrow M^{M} \otimes$ $\mathcal{W}_{D^{m+n}}$ for the morphism obtained as the composition of

$$
\begin{aligned}
& \left(\operatorname{id}_{M^{M}} \otimes \mathcal{W}_{\left.\left(d_{1}, \ldots, d_{m}, d_{m+1}, \ldots, d_{m+n}\right) \in D^{m+n_{\mapsto}}\left(d_{1}, \ldots, d_{m}\right) \in D^{m}\right) \times}\right. \\
& \left(\operatorname{id}_{M^{M}} \otimes \mathcal{W}_{\left(d_{1}, \ldots, d_{m}, d_{m+1}, \ldots, d_{m+n}\right) \in D^{m+n} \mapsto\left(d_{m+1}, \ldots, d_{m+n}\right) \in D^{n}}\right) \\
& :\left(M^{M} \otimes \mathcal{W}_{D^{m}}\right) \times\left(M^{M} \otimes \mathcal{W}_{D^{n}}\right) \rightarrow\left(M^{M} \otimes \mathcal{W}_{D^{m+n}}\right) \times\left(M^{M} \otimes \mathcal{W}_{D^{m+n}}\right) \\
& =\left(M^{M} \times M^{M}\right) \otimes \mathcal{W}_{D^{m+n}} \\
& \operatorname{ass}_{M} \otimes \operatorname{id}_{\mathcal{W}_{D^{m+n}}}:\left(M^{M} \times M^{M}\right) \otimes \mathcal{W}_{D^{m+n}} \rightarrow M^{M} \otimes \mathcal{W}_{D^{m+n}}
\end{aligned}
$$

in succession.

Lemma 4.7. We have such laws of associativity as

$$
\begin{aligned}
& \operatorname{ass}_{M} \circ\left(\operatorname{ass}_{M} \times \operatorname{id}_{M^{M}}\right)=\operatorname{ass}_{M} \circ\left(\operatorname{id}_{M^{M}} \times \operatorname{ass}_{M}\right), \\
& \overline{\operatorname{ass}}_{M} \circ\left(\overline{\operatorname{ass}}_{M} \times \operatorname{id}_{M^{M}}\right)=\overline{\operatorname{ass}}_{M} \circ\left(\operatorname{id}_{M^{M}} \times \overline{\operatorname{ass}}_{M}\right) .
\end{aligned}
$$

Proposition 4.8. We have such laws of associativity as follows:

$$
\begin{aligned}
& \operatorname{Ass}_{M}^{l+m, n} \circ\left(\operatorname{Ass}_{M}^{l, m} \times \operatorname{id}_{M^{M} \otimes \mathcal{W}_{D^{n}}}\right) \\
= & \operatorname{Ass}_{M}^{l, m+n} \circ\left(\operatorname{id}_{M^{M} \otimes \mathcal{W}_{D^{l}}} \times \operatorname{Ass}_{M}^{m, n}\right)
\end{aligned}
$$

and

$$
\begin{aligned}
& \overline{\operatorname{Ass}}_{M}^{l+m, n} \circ\left(\overline{\operatorname{Ass}}_{M}^{l, m} \times \mathrm{id}_{M^{M} \otimes \mathcal{W}_{D^{n}}}\right) \\
= & \overline{\operatorname{Ass}}_{M}^{l, m+n} \circ\left(\operatorname{id}_{M^{M} \otimes \mathcal{W}_{D^{l}}} \times \overline{\operatorname{Ass}}_{M}^{m, n}\right) .
\end{aligned}
$$

Proof. Here we deal only with the former, leaving the latter to the reader. Now we have to show that the composition of

$$
\begin{aligned}
& \operatorname{Ass}_{M}^{l, m} \times \operatorname{id}_{M^{M} \otimes \mathcal{W}_{D^{n}}}:\left(M^{M} \otimes \mathcal{W}_{D^{l}}\right) \times\left(M^{M} \otimes \mathcal{W}_{D^{m}}\right) \times\left(M^{M} \otimes \mathcal{W}_{D^{n}}\right) \\
= & \left(\left(M^{M} \otimes \mathcal{W}_{D^{l}}\right) \times\left(M^{M} \otimes \mathcal{W}_{D^{m}}\right)\right) \times\left(M^{M} \otimes \mathcal{W}_{D^{n}}\right) \\
\rightarrow & \left(M^{M} \otimes \mathcal{W}_{D^{l+m}}\right) \times\left(M^{M} \otimes \mathcal{W}_{D^{n}}\right)
\end{aligned}
$$

followed by

$$
\operatorname{Ass}_{M}^{l+m, n}:\left(M^{M} \otimes \mathcal{W}_{D^{l+m}}\right) \times\left(M^{M} \otimes \mathcal{W}_{D^{n}}\right) \rightarrow M^{M} \otimes \mathcal{W}_{D^{l+m+n}}
$$


is equal to that of

$$
\begin{aligned}
& \operatorname{id}_{M^{M} \otimes \mathcal{W}_{D^{l}}} \times \operatorname{Ass}_{M}^{m, n}:\left(M^{M} \otimes \mathcal{W}_{D^{l}}\right) \times\left(M^{M} \otimes \mathcal{W}_{D^{m}}\right) \times\left(M^{M} \otimes \mathcal{W}_{D^{n}}\right) \\
= & \left(M^{M} \otimes \mathcal{W}_{D^{l}}\right) \times\left(\left(M^{M} \otimes \mathcal{W}_{D^{m}}\right) \times\left(M^{M} \otimes \mathcal{W}_{D^{n}}\right)\right) \\
\rightarrow & \left(M^{M} \otimes \mathcal{W}_{D^{l}}\right) \times\left(M^{M} \otimes \mathcal{W}_{D^{m+n}}\right)
\end{aligned}
$$

followed by

$$
\operatorname{Ass}_{M}^{l, m+n}:\left(M^{M} \otimes \mathcal{W}_{D^{l}}\right) \times\left(M^{M} \otimes \mathcal{W}_{D^{m+n}}\right) \rightarrow M^{M} \otimes \mathcal{W}_{D^{l+m+n}} .
$$

The former is the composition of

$$
\begin{aligned}
& \left(M^{M} \otimes \mathcal{W}_{D^{l}}\right) \times\left(M^{M} \otimes \mathcal{W}_{D^{m}}\right) \times\left(M^{M} \otimes \mathcal{W}_{D^{n}}\right) \\
= & \left(\left(M^{M} \otimes \mathcal{W}_{D^{l}}\right) \times\left(M^{M} \otimes \mathcal{W}_{D^{m}}\right)\right) \times\left(M^{M} \otimes \mathcal{W}_{D^{n}}\right) \\
\rightarrow & \left(\left(M^{M} \otimes \mathcal{W}_{D^{l+m}}\right) \times\left(M^{M} \otimes \mathcal{W}_{D^{l+m}}\right)\right) \times\left(M^{M} \otimes \mathcal{W}_{D^{n}}\right) \\
= & \left(\left(M^{M} \times M^{M}\right) \otimes \mathcal{W}_{D^{l+m}}\right) \times\left(M^{M} \otimes \mathcal{W}_{D^{n}}\right),
\end{aligned}
$$

$\left(\operatorname{ass}_{M} \otimes \operatorname{id}_{\mathcal{W}_{D^{l+m}}}\right) \times \operatorname{id}_{M^{M} \otimes \mathcal{W}_{D^{n}}}:\left(\left(M^{M} \times M^{M}\right) \otimes \mathcal{W}_{D^{l+m}}\right) \times\left(M^{M} \otimes \mathcal{W}_{D^{n}}\right)$

$$
\rightarrow\left(M^{M} \otimes \mathcal{W}_{D^{l+m}}\right) \times\left(M^{M} \otimes \mathcal{W}_{D^{n}}\right)
$$

$$
\begin{aligned}
& \left(M^{M} \otimes \mathcal{W}_{D^{l+m}}\right) \times\left(M^{M} \otimes \mathcal{W}_{D^{n}}\right) \\
\rightarrow & \left(M^{M} \otimes \mathcal{W}_{D^{l+m+n}}\right) \times\left(M^{M} \otimes \mathcal{W}_{D^{l+m+n}}\right) \\
= & \left(M^{M} \times M^{M}\right) \otimes \mathcal{W}_{D^{l+m+n}}
\end{aligned}
$$

and

$$
\operatorname{ass}_{M} \otimes \operatorname{id}_{\mathcal{W}_{D^{l+m+n}}}:\left(M^{M} \times M^{M}\right) \otimes \mathcal{W}_{D^{l+m+n}} \rightarrow M^{M} \otimes \mathcal{W}_{D^{l+m+n}}
$$

in succession, while the latter is the composition of

$$
\begin{aligned}
&\left(M^{M} \otimes \mathcal{W}_{D^{l}}\right) \times\left(M^{M} \otimes \mathcal{W}_{D^{m}}\right) \times\left(M^{M} \otimes \mathcal{W}_{D^{n}}\right) \\
&=\left(M^{M} \otimes \mathcal{W}_{D^{l}}\right) \times\left(\left(M^{M} \otimes \mathcal{W}_{D^{m}}\right) \times\left(M^{M} \otimes \mathcal{W}_{D^{n}}\right)\right) \\
& \rightarrow\left(M^{M} \otimes \mathcal{W}_{D^{l}}\right) \times\left(\left(M^{M} \otimes \mathcal{W}_{D^{m+n}}\right) \times\left(M^{M} \otimes \mathcal{W}_{D^{m+n}}\right)\right) \\
&=\left(M^{M} \otimes \mathcal{W}_{D^{l}}\right) \times\left(\left(M^{M} \times M^{M}\right) \otimes \mathcal{W}_{D^{m+n}}\right), \\
& \operatorname{id}_{M^{M} \otimes \mathcal{W}_{D^{l}}} \times\left(\operatorname{ass}_{M} \otimes \operatorname{id}_{\mathcal{W}_{D^{m+n}}}\right):\left(M^{M} \otimes \mathcal{W}_{D^{l}}\right) \times\left(\left(M^{M} \times M^{M}\right) \otimes \mathcal{W}_{D^{m+n}}\right) \\
& \rightarrow\left(M^{M} \otimes \mathcal{W}_{D^{l}}\right) \times\left(M^{M} \otimes \mathcal{W}_{D^{m+n}}\right), \\
& \\
&\left(M^{M} \otimes \mathcal{W}_{D^{l+m}}\right) \times\left(M^{M} \otimes \mathcal{W}_{D^{n}}\right) \\
& \rightarrow\left(M^{M} \otimes \mathcal{W}_{D^{l+m+n}}\right) \times\left(M^{M} \otimes \mathcal{W}_{D^{l+m+n}}\right) \\
&=\left(M^{M} \times M^{M}\right) \otimes \mathcal{W}_{D^{l+m+n}}
\end{aligned}
$$


and (4.5) in succession. Since the composition of (4.3) and (4.4) in succession is equal to that of

$$
\begin{aligned}
& \left(\left(M^{M} \times M^{M}\right) \otimes \mathcal{W}_{D^{l+m}}\right) \times\left(M^{M} \otimes \mathcal{W}_{D^{n}}\right) \\
\rightarrow & \left(\left(M^{M} \times M^{M}\right) \otimes \mathcal{W}_{D^{l+m+n}}\right) \times\left(M^{M} \otimes \mathcal{W}_{D^{l+m+n}}\right) \\
= & \left(\left(M^{M} \times M^{M}\right) \times M^{M}\right) \otimes \mathcal{W}_{D^{l+m+n}}
\end{aligned}
$$

and

$$
\begin{aligned}
& \left(\operatorname{ass}_{M} \times \operatorname{id}_{M^{M}}\right) \otimes \operatorname{id}_{\mathcal{W}_{D^{l+m+n}}}:\left(\left(M^{M} \times M^{M}\right) \times M^{M}\right) \otimes \mathcal{W}_{D^{l+m+n}} \\
\rightarrow & \left(M^{M} \times M^{M}\right) \otimes \mathcal{W}_{D^{l+m+n}}
\end{aligned}
$$

in succession, we conclude that the left-hand side of (4.1) is equal to the composition of (4.2), (4.6), (4.7)and (4.5) in succession, which is in turn equal to the composition of

$$
\begin{aligned}
& \left(M^{M} \otimes \mathcal{W}_{D^{l}}\right) \times\left(M^{M} \otimes \mathcal{W}_{D^{m}}\right) \times\left(M^{M} \otimes \mathcal{W}_{D^{n}}\right) \\
\rightarrow & \left(M^{M} \otimes \mathcal{W}_{D^{l+m+n}}\right) \times\left(M^{M} \otimes \mathcal{W}_{D^{l+m+n}}\right) \times\left(M^{M} \otimes \mathcal{W}_{D^{l+m+n}}\right) \\
= & \left(M^{M} \times M^{M} \times M^{M}\right) \otimes \mathcal{W}_{D^{l+m+n}} \\
= & \left(\left(M^{M} \times M^{M}\right) \times M^{M}\right) \otimes \mathcal{W}_{D^{l+m+n}},
\end{aligned}
$$

(4.7) and (4.5) in succession. Similarly, the right-hand side of (4.1) is the composition of

$$
\begin{aligned}
& \left(M^{M} \otimes \mathcal{W}_{D^{l}}\right) \times\left(M^{M} \otimes \mathcal{W}_{D^{m}}\right) \times\left(M^{M} \otimes \mathcal{W}_{D^{n}}\right) \\
\rightarrow & \left(M^{M} \otimes \mathcal{W}_{D^{l+m+n}}\right) \times\left(M^{M} \otimes \mathcal{W}_{D^{l+m+n}}\right) \times\left(M^{M} \otimes \mathcal{W}_{D^{l+m+n}}\right) \\
= & \left(M^{M} \times M^{M} \times M^{M}\right) \otimes \mathcal{W}_{D^{l+m+n}} \\
= & \left(M^{M} \times\left(M^{M} \times M^{M}\right)\right) \otimes \mathcal{W}_{D^{l+m+n}}, \\
& \left(\operatorname{id}_{M^{M}} \times \operatorname{ass}_{M}\right) \otimes \operatorname{id}_{\mathcal{W}_{D^{l+m+n}}}:\left(M^{M} \times\left(M^{M} \times M^{M}\right)\right) \otimes \mathcal{W}_{D^{l+m+n}} \\
\rightarrow & \left(M^{M} \times M^{M}\right) \otimes \mathcal{W}_{D^{l+m+n}}
\end{aligned}
$$

and (4.5) in succession. Therefore the desired result follows from Lemma 4.7.

Remark 4.9. By the above associativity, we can unambiguously define such a morphism as

$$
\begin{aligned}
& \operatorname{Ass}_{M}^{1,1,1}:\left(M^{M} \otimes \mathcal{W}_{D}\right) \times\left(M^{M} \otimes \mathcal{W}_{D}\right) \times\left(M^{M} \otimes \mathcal{W}_{D}\right) \\
\rightarrow & M^{M} \otimes \mathcal{W}_{D^{3}}
\end{aligned}
$$

to be either the composition of

$$
\begin{aligned}
& \operatorname{Ass}_{M}^{1,1} \times \operatorname{id}_{M^{M} \otimes \mathcal{W}_{D}}:\left(M^{M} \otimes \mathcal{W}_{D}\right) \times\left(M^{M} \otimes \mathcal{W}_{D}\right) \times\left(M^{M} \otimes \mathcal{W}_{D}\right) \\
= & \left(\left(M^{M} \otimes \mathcal{W}_{D}\right) \times\left(M^{M} \otimes \mathcal{W}_{D}\right)\right) \times\left(M^{M} \otimes \mathcal{W}_{D}\right) \\
\rightarrow & \left(M^{M} \otimes \mathcal{W}_{D^{2}}\right) \times\left(M^{M} \otimes \mathcal{W}_{D}\right)
\end{aligned}
$$


and

$$
\begin{aligned}
& \operatorname{Ass}_{M}^{2,1}:\left(M^{M} \otimes \mathcal{W}_{D^{2}}\right) \times\left(M^{M} \otimes \mathcal{W}_{D}\right) \\
\rightarrow & M^{M} \otimes \mathcal{W}_{D^{3}}
\end{aligned}
$$

in succession, or the composition of

$$
\begin{aligned}
& \operatorname{id}_{M^{M} \otimes \mathcal{W}_{D}} \times \operatorname{Ass}_{M}^{1,1}:\left(M^{M} \otimes \mathcal{W}_{D}\right) \times\left(M^{M} \otimes \mathcal{W}_{D}\right) \times\left(M^{M} \otimes \mathcal{W}_{D}\right) \\
= & \left(M^{M} \otimes \mathcal{W}_{D}\right) \times\left(\left(M^{M} \otimes \mathcal{W}_{D}\right) \times\left(M^{M} \otimes \mathcal{W}_{D}\right)\right) \\
\rightarrow & \left(M^{M} \otimes \mathcal{W}_{D}\right) \times\left(M^{M} \otimes \mathcal{W}_{D^{2}}\right)
\end{aligned}
$$

and

$$
\begin{aligned}
& \operatorname{Ass}_{M}^{1,2}:\left(M^{M} \otimes \mathcal{W}_{D}\right) \times\left(M^{M} \otimes \mathcal{W}_{D^{2}}\right) \\
\rightarrow & M^{M} \otimes \mathcal{W}_{D^{3}}
\end{aligned}
$$

in succession. Similarly for $\overline{\operatorname{Ass}}_{M}^{1,1,1}$.

Notation 4.10. We write $i_{M}: 1 \rightarrow M^{M}$ for the exponential transpose of

$$
\operatorname{id}_{M}: 1 \times M=M \rightarrow M \text {. }
$$

Lemma 4.11. We have

$$
\operatorname{ass}_{M} \circ\left(i_{M} \times \operatorname{id}_{M^{M}}\right)=\operatorname{ass}_{M} \circ\left(\operatorname{id}_{M^{M}} \times i_{M}\right)=\operatorname{id}_{M^{M}}
$$

and

$$
\overline{\operatorname{ass}}_{M} \circ\left(i_{M} \times \operatorname{id}_{M^{M}}\right)=\overline{\operatorname{ass}}_{M} \circ\left(\operatorname{id}_{M^{M}} \times i_{M}\right)=\operatorname{id}_{M^{M}} .
$$

Notation 4.12. We write $I_{M}^{n}: 1 \rightarrow M^{M} \otimes \mathcal{W}_{D^{n}}$ for the morphism

$$
i_{M} \otimes \operatorname{id}_{\mathcal{W}_{D^{n}}}: 1=1 \otimes \mathcal{W}_{D^{n}} \rightarrow M^{M} \otimes \mathcal{W}_{D^{n}} .
$$

Proposition 4.13. We have such identities as

$$
\begin{aligned}
& \operatorname{Ass}_{M}^{m, n} \circ\left(I_{M}^{m} \times \operatorname{id}_{M^{M} \otimes \mathcal{W}_{D^{n}}}\right) \\
= & \operatorname{id}_{M^{M}} \otimes \mathcal{W}_{\left(d_{1}, \ldots, d_{m}, d_{m+1}, \ldots, d_{m+n}\right) \in D^{m+n} \mapsto\left(d_{m+1}, \ldots, d_{m+n}\right) \in D^{n}} \\
& \operatorname{Ass}_{M}^{m, n} \circ\left(\operatorname{id}_{\left.M^{M} \otimes \mathcal{W}_{D^{m}} \times I_{M}^{n}\right)}\right) \\
= & \operatorname{id}_{M^{M}} \otimes \mathcal{W}_{\left(d_{1}, \ldots, d_{m}, d_{m+1}, \ldots, d_{m+n}\right) \in D^{m+n} \mapsto\left(d_{1}, \ldots, d_{m}\right) \in D^{m}} \\
& \overline{\operatorname{Ass}}_{M}^{m, n} \circ\left(I_{M}^{m} \times \operatorname{id}_{M^{M} \otimes \mathcal{W}_{D^{n}}}\right) \\
= & \operatorname{id}_{M^{M}} \otimes \mathcal{W}_{\left(d_{1}, \ldots, d_{m}, d_{m+1}, \ldots, d_{m+n}\right) \in D^{m+n} \mapsto\left(d_{m+1}, \ldots, d_{m+n}\right) \in D^{n}}
\end{aligned}
$$

and

$$
\begin{aligned}
& \overline{\operatorname{Ass}}_{M}^{m, n} \circ\left(\operatorname{id}_{M^{M} \otimes \mathcal{W}_{D^{m}}} \times I_{M}^{n}\right) \\
= & \operatorname{id}_{M^{M}} \otimes \mathcal{W}_{\left(d_{1}, \ldots, d_{m}, d_{m+1}, \ldots, d_{m+n}\right) \in D^{m+n_{\mapsto}} \rightarrow\left(d_{1}, \ldots, d_{m}\right) \in D^{m} .}
\end{aligned}
$$

Proof. These follow from Lemma 4.11 by the same token as in the proof Proposition 4.8 .

The following proposition is essentially a variant of Proposition 6 in $\S 3.2$ of [2]. 
Proposition 4.14. The composition of

$$
\begin{aligned}
& \left(M^{M} \otimes \mathcal{W}_{D}\right)_{\mathrm{id}_{M}} \times\left(M^{M} \otimes \mathcal{W}_{D}\right)_{\mathrm{id}_{M}} \rightarrow\left(M^{M} \otimes \mathcal{W}_{D}\right) \times\left(M^{M} \otimes \mathcal{W}_{D}\right), \\
& \operatorname{Ass}_{M}^{1,1}:\left(M^{M} \otimes \mathcal{W}_{D}\right) \times\left(M^{M} \otimes \mathcal{W}_{D}\right) \rightarrow M^{M} \otimes \mathcal{W}_{D^{2}}
\end{aligned}
$$

and

$$
M^{M} \otimes \mathcal{W}_{D^{2}} \rightarrow M^{M} \otimes \mathcal{W}_{D(2)}
$$

in succession is equal to the canonical injection

$$
\left(M^{M} \otimes \mathcal{W}_{D}\right)_{\mathrm{id}_{M}} \times\left(M^{M} \otimes \mathcal{W}_{D}\right)_{\mathrm{id}_{M}} \rightarrow M^{M} \otimes \mathcal{W}_{D(2)}
$$

Similarly, the composition of (4.8),

$$
\overline{\operatorname{Ass}}_{M}^{1,1}:\left(M^{M} \otimes \mathcal{W}_{D}\right) \times\left(M^{M} \otimes \mathcal{W}_{D}\right) \rightarrow M^{M} \otimes \mathcal{W}_{D^{2}}
$$

and (4.9) in succession is equal to (4.10).

Proof. Here we deal only with the former, leaving a similar treatment of the latter to the reader. It suffices to show that the composition of (4.8)-(4.9) and

$$
\operatorname{id}_{M^{M}} \otimes \mathcal{W}_{d \in D \mapsto(d, 0) \in D(2)}: M^{M} \otimes \mathcal{W}_{D(2)} \rightarrow M^{M} \otimes \mathcal{W}_{D}
$$

in succession is equal to the composition of the projection to the first factor

$$
\left(M^{M} \otimes \mathcal{W}_{D}\right)_{\mathrm{id}_{M}} \times\left(M^{M} \otimes \mathcal{W}_{D}\right)_{\mathrm{id}_{M}} \rightarrow\left(M^{M} \otimes \mathcal{W}_{D}\right)_{\mathrm{id}_{M}}
$$

followed by the canonical injection

$$
\left(M^{M} \otimes \mathcal{W}_{D}\right)_{\mathrm{id}_{M}} \rightarrow M^{M} \otimes \mathcal{W}_{D}
$$

while the composition of (4.8)-(4.9) and

$$
\operatorname{id}_{M^{M}} \otimes \mathcal{W}_{d \in D \mapsto(0, d) \in D(2)}: M^{M} \otimes \mathcal{W}_{D(2)} \rightarrow M^{M} \otimes \mathcal{W}_{D}
$$

in succession is equal to the composition of the projection of the second factor

$$
\left(M^{M} \otimes \mathcal{W}_{D}\right)_{\mathrm{id}_{M}} \times\left(M^{M} \otimes \mathcal{W}_{D}\right)_{\mathrm{id}_{M}} \rightarrow\left(M^{M} \otimes \mathcal{W}_{D}\right)_{\mathrm{id}_{M}}
$$

followed by the canonical injection

$$
\left(M^{M} \otimes \mathcal{W}_{D}\right)_{\operatorname{id}_{M}} \rightarrow M^{M} \otimes \mathcal{W}_{D}
$$

the details of which are left to the reader.

Corollary 4.15. The composition of (4.8)-(4.9) followed by

$$
\operatorname{id}_{M^{M}} \otimes \mathcal{W}_{d \in D \mapsto(d, d) \in D(2)}: M^{M} \otimes \mathcal{W}_{D(2)} \rightarrow M^{M} \otimes \mathcal{W}_{D}
$$

is factored uniquely into

$$
\left(M^{M} \otimes \mathcal{W}_{D}\right)_{\mathrm{id}_{M}} \times\left(M^{M} \otimes \mathcal{W}_{D}\right)_{\mathrm{id}_{M}} \rightarrow\left(M^{M} \otimes \mathcal{W}_{D}\right)_{\mathrm{id}_{M}}
$$

followed by the canonical morphism

$$
\left(M^{M} \otimes \mathcal{W}_{D}\right)_{\mathrm{id}_{M}} \rightarrow M^{M} \otimes \mathcal{W}_{D}
$$

The arrow in (4.11) stands for the addition of the $k$-module $\left(M^{M} \otimes \mathcal{W}_{D}\right)_{\mathrm{id}_{M}}$. 
Lemma 4.16. The diagram

$$
\mathcal{W}_{D} \rightarrow \mathcal{W}_{D^{2}} \rightarrow \mathcal{W}_{D}
$$

is a limit diagram in $\mathcal{W}$ eil $l_{k}$, where the left horizontal arrow is

$$
\mathcal{W}_{\left(d_{1}, d_{2}\right) \in D^{2} \mapsto d_{1} d_{2} \in D}
$$

while the three right horizontal arrows are

$$
\begin{aligned}
& \mathcal{W}_{d \in D \mapsto(d, 0) \in D^{2}} \\
& \mathcal{W}_{d \in D \mapsto(0, d) \in D^{2}} \\
& \mathcal{W}_{d \in D \mapsto(0,0) \in D^{2}} .
\end{aligned}
$$

Proof. The reader is referred to Proposition 7 in $\S 2.2$ of [2].

Theorem 4.17. The diagram

$$
\begin{aligned}
M^{M} \otimes \mathcal{W}_{D} \rightarrow & \rightarrow & \rightarrow \\
M^{M} \otimes \mathcal{W}_{D^{2}} & \rightarrow & \\
& \rightarrow & \\
\left(M^{M} \otimes \mathcal{W}_{D}\right)_{\mathrm{id}_{M}} \times\left(M^{M} \otimes \mathcal{W}_{D}\right)_{\mathrm{id}_{M}} & &
\end{aligned}
$$

is commutative, where the left horizontal arrow is

$$
\operatorname{id}_{M^{M}} \otimes \mathcal{W}_{\left(d_{1}, d_{2}\right) \in D^{2} \mapsto d_{1} d_{2} \in D}
$$

the three right horizontal arrows are

$$
\begin{aligned}
& \operatorname{id}_{M^{M}} \otimes \mathcal{W}_{d \in D \mapsto(d, 0) \in D^{2}} \\
& \operatorname{id}_{M^{M}} \otimes \mathcal{W}_{d \in D \mapsto(0, d) \in D^{2}} \\
& \operatorname{id}_{M^{M}} \otimes \mathcal{W}_{d \in D \mapsto(0,0) \in D^{2}}
\end{aligned}
$$

and the vertical arrow

$$
\left(M^{M} \otimes \mathcal{W}_{D}\right)_{\mathrm{id}_{M}} \times\left(M^{M} \otimes \mathcal{W}_{D}\right)_{\mathrm{id}_{M}} \rightarrow M^{M} \otimes \mathcal{W}_{D^{2}}
$$

is the composition of

$$
\begin{aligned}
\left(M^{M} \otimes \mathcal{W}_{D}\right)_{\mathrm{id}_{M}} \times\left(M^{M} \otimes \mathcal{W}_{D}\right)_{\mathrm{id}_{M}} \rightarrow\left(M^{M} \otimes \mathcal{W}_{D}\right) \times\left(M^{M} \otimes \mathcal{W}_{D}\right), \\
\quad\left\langle p r_{1}, p r_{2}, p r_{1}, p r_{2}\right\rangle:\left(M^{M} \otimes \mathcal{W}_{D}\right) \times\left(M^{M} \otimes \mathcal{W}_{D}\right) \\
\rightarrow\left(M^{M} \otimes \mathcal{W}_{D}\right) \times\left(M^{M} \otimes \mathcal{W}_{D}\right) \times\left(M^{M} \otimes \mathcal{W}_{D}\right) \times\left(M^{M} \otimes \mathcal{W}_{D}\right), \\
\operatorname{Ass}_{M}^{1,1,1,1}:\left(M^{M} \otimes \mathcal{W}_{D}\right) \times\left(M^{M} \otimes \mathcal{W}_{D}\right) \times\left(M^{M} \otimes \mathcal{W}_{D}\right) \times\left(M^{M} \otimes \mathcal{W}_{D}\right) \\
\rightarrow M^{M} \otimes \mathcal{W}_{D^{4}}
\end{aligned}
$$

and

$$
\operatorname{id}_{M^{M}} \otimes \mathcal{W}_{\left(d_{1}, d_{2}\right) \in D^{2} \mapsto\left(d_{1}, d_{2},-d_{1},-d_{2}\right) \in D^{4}}: M^{M} \otimes \mathcal{W}_{D^{4}} \rightarrow M^{M} \otimes \mathcal{W}_{D^{2}}
$$

in succession. Therefore, there exists a unique arrow

$$
\left(M^{M} \otimes \mathcal{W}_{D}\right)_{\mathrm{id}_{M}} \times\left(M^{M} \otimes \mathcal{W}_{D}\right)_{\mathrm{id}_{M}} \rightarrow M^{M} \otimes \mathcal{W}_{D}
$$


making the triangle

$$
\begin{aligned}
& M^{M} \otimes \mathcal{W}_{D} \rightarrow \quad M^{M} \otimes \mathcal{W}_{D^{2}} \\
& \left(M^{M} \otimes \mathcal{W}_{D}\right)_{\mathrm{id}_{M}} \times\left(M^{M} \otimes \mathcal{W}_{D}\right)_{\mathrm{id}_{M}}
\end{aligned}
$$

commutative. Furthermore, the arrow in (4.15) is factored uniquely into

$$
\left(M^{M} \otimes \mathcal{W}_{D}\right)_{\mathrm{id}_{M}} \times\left(M^{M} \otimes \mathcal{W}_{D}\right)_{\mathrm{id}_{M}} \rightarrow\left(M^{M} \otimes \mathcal{W}_{D}\right)_{\mathrm{id}_{M}}
$$

followed by

$$
\left(M^{M} \otimes \mathcal{W}_{D}\right)_{\operatorname{id}_{M}} \rightarrow M^{M} \otimes \mathcal{W}_{D}
$$

Proof. It is easy to see that the composition of the arrow in (4.14) and any one of the three arrows in (4.12)-(4.13) results in the same arrow. Therefore the second statement follows directly from Lemma 4.16. The last statement is easy to verify.

Notation 4.18. The arrow in (4.16) is denoted by $L_{M}$.

Theorem 4.19. The $k$-module $\left(M^{M} \otimes \mathcal{W}_{D}\right)_{\mathrm{id}_{M}}$ endowed with $L_{M}$ as a Lie bracket is a Lie $k$-algebra object in the category $\mathcal{K}$.

Proof. The proof could be obtained by reformulating our proof of Proposition 16 above and Sections 5 and 6 in [3]. The Jacobi identity is dealt with in detail in $[5]$.

\section{RefERENCES}

[1] A. Kock, Synthetic Differential Geometry, 2nd ed., Cambridge University Press, Cambridge, 2006.

[2] R. Lavendhomme, Basic Concepts of Synthetic Differential Geometry, Kluwer, Dordrecht, 1996.

[3] H. Nishimura, Differential geometry of microlinear Frölicher spaces I, IJPAM 64 (2010), 43-83.

[4] H. Nishimura, Axiomatic differential geometry I -towards model categories of differential geometry-, arXiv 1203.3911.

[5] H. Nishimura, Axiomatic differential geometry II-3 -its developments- chapter 3: the general Jacobi identity, arXiv 1208.1894.

Hirokazu Nishimura, Institute of Mathematics, University of Tsukuba, Tsukuba, Ibaraki, 305-8571, Japan

e-mail: logic@math.tsukuba.ac.jp 
